\title{
A Corpus-Driven Study of Chinese Children's Multimodal Refusal and Its Implications for Home Education
}

\author{
Rongbin Wang, Pei Wang \\ School of Foreign Languages, Shanxi University, Taiyuan, China \\ Email: wangrongbin@sxu.edu.cn,1095915405@qq.com
}

How to cite this paper: Wang, R. B., \& Wang, P. (2019). A Corpus-Driven Study of Chinese Children's Multimodal Refusal and Its Implications for Home Education. Open Journal of Modern Linguistics, 9, 317-329. https://doi.org/10.4236/ojml.2019.95026

Received: June 20, 2018

Accepted: September 8, 2019

Published: September 11, 2019

Copyright (อ 2019 by author(s) and Scientific Research Publishing Inc. This work is licensed under the Creative Commons Attribution International License (CC BY 4.0).

http://creativecommons.org/licenses/by/4.0/

\section{(c) (i) Open Access}

\begin{abstract}
Multimodal refusal is a common type of multimodal communicative act. Driven by naturally occurred corpus, this paper first classifies the parental acts that usually trigger children's refusal into three types: positive parenting, negative parenting and non-parenting acts. Then the paper thoroughly analyzes the multimodal representational characteristics of Chinese-speaking children's refusal and classifies their multimodal refusal acts according to the modal involvement on the one hand and the semantic degree of refusal on the other hand. They are verbal-dominant refusal, nonverbal-dominant refusal and verbal-nonverbal consistent refusal according to modal involvement and complete refusal and partial refusal according to degree of refusal. Finally, the paper summarizes three pieces of suggestions for parents in home education, including emphasizing the multimodal nature of children's refusal, adjusting parenting acts that may trigger children's refusal and cultivating children's ability of multimodal refusal.
\end{abstract}

\section{Keywords}

Chinese-Speaking Children, Multimodal Refusal, Corpus-Driven, Parenting, Home Education

\section{Introduction}

In recent years, Chinese educational institution has paid more and more attention to the scientific construction and development of pre-school education and primary school education. As the General Secretary Xi Jinping once stated in the reports of the $19^{\text {th }}$ National Congress, it is a must for the country to develop a systematic pre-school education fully and build it into a high-quality education. 
Similarly, in the governmental work reports of the Two Sessions in 2019, the Premier Li Keqiang also pointed out that elementary education should be organized more fairly and superbly.

Home education is firstly, "full-time education of children in and around the home by their parents or guardians and secondly", "where the parents are committed to their children's education and home-educating" (Petrie, 1998). While a high-quality pre-school education involves many aspects, one of the key factors is to ensure that children receive a scientific and appropriate home education before school because home education is the first lesson of life education as well as the foundation of school education and social education (Zhai, 2016). For instance, it plays an important role in fostering children's sound personality, living and learning habits and their necessary sociability. The Guidelines of the National Program for Medium and Long-term Educational Reform and Development (2010-2020) clearly presents the critical role and effect of home education in Chinese education reform and development (Cares about the Next Generation Work Committee at Ministry of Education, 2012).

Refusal is a commonly occurred communicative act. Besides verbal expression, a communicative act like refusal can also be realized through nonverbal semiotic resources (Austin, 1979). To be specific, multimodal refusal in this study refers to the acts in which Chinese children as interlocutors make use of both verbal and nonverbal resources, that is, language on the one hand and facial expression, gesture, and posture etc. on the other hand, to express different meanings of refusal, or in other words, conduct multimodal acts of refusal. In the context of home education, the initiation of children's multimodal refusal is not accidental, but is a response made by children who are influenced by specific communicative factors, among which parenting toward children is a key factor. In many cases, children's multimodal refusal is their feedback to parental acts of home education and reflects the degree of children's acceptance toward home education.

Based on a naturally occurred corpus of 3- to 8-year-old Chinese children's multimodal refusal, this paper explores the multimodal characteristics of children's acts of refusal triggered by parenting with the help of a corpus-driven analytical approach combined with a quantitative analysis. To begin with, the paper endeavors to analyze and classify the parenting acts in corpus, and then examines different ways of multimodal representation of children's refusal toward different parenting acts so as to find out the communicative characteristics of Chinese-speaking children, especially those characteristics related to their multimodal acts of refusal, hoping to provide some useful enlightenment for the home education to Chinese children.

\section{Literature Review}

The word "refusal" was defined as "an act of saying or showing that you will not do, give or accept something" in the Oxford Advanced Learner's Eng- 
lish-Chinese Dictionary (6 ${ }^{\text {th }}$ Edition). Searle (1975) put forward the indirect speech act theory consisting of five types of illocutionary act, according to which refusal was a kind of commissive act. Brown \& Levison (1987) pointed out that refusal was a face-threatening act, which damaged the positive face of the hearer. Turnbull (1992) described refusal as dispreferred second pair in contrast to request-compliance, which was the preferred second pair. Chen et al. (1998) defined refusal as a speech act in which a speaker "denies to engage in an action proposed by the interlocutor".

As was shown above, pervious researches commonly regarded refusal as a dispreferred and face-threatening act. As for multimodal behavior of children, some researchers mainly concerned about gestures of those with autism spectrum disorders, other researchers paid attention to the cognitive development of children by observing their language and gesture change as they grew up.

To narrow down, researches on the multimodal rejections from the perspective of Systemic Functional Linguistics mainly paid attention to the use of gestures. For example, Guidetti \& Michele (2000) studied the change of gesture and language forms of French-speaking children when expressing agreement and rejection and found that the information forms of children changed with their age. Guidetti \& Michele (2005) studied the forms and function of the rejection of French-speaking children and found that language, gesture, and the combination of language and gesture were all forms of rejection and the major function of refusal was to determine. Liang Biying (2007) analyzed the relationship between the meaning of "advances" and "refusal" and the pure function of language from the perspective of Systemic Functional Linguistics. Bressem \& Müller (2014) studied the set of gestures used by Germans in expressing denial, refusal and negative comments. They were sweeping away, holding away, throwing away and brushing away. Bressem et al. (2017) analyzed two repeated gestures in German: sweeping away and holding away. This research showed that the semantic core of these two gestures is consistent with their specific referential meaning and pragmatic meaning.

These studies above mainly focused on the role of non-verbal modes in the realization of meaning of rejection by using empirical, qualitative and quantitative research methods. However, non-verbal refusal was far less studied than verbal refusal. Therefore, there was still large space for research.

\section{Theoretical Framework}

This study mainly follows the idea of Systemic Functional Linguistics and Social Semiotics, according to which meaning-making is a systemic making of linguistic choices from system, which includes three aspects: 1) the "entry condition" (where the choice is made), 2) the set of possible options and 3) the "realizations" (Halliday, 1994). In this section, an overview of the social semiotic framework will be given, which is followed by a development of an analytical modal of children's refusal. 
In Social Semiotics, context is conceptualized as "the total environment in which a text unfolds" (Halliday, 1978). Context is stratified into "Context of Situation" and "Context of Culture". The "Context of Situation" includes three dimensions: Field, Tenor and Mode. Field refers to the nature of the social action that is taking place. Tenor deals with the participants taking part and the nature of the relationship among them in terms of power and distance. Mode is concerned with the channel of communication which consists of Medium (e.g. face-to-face, print media) and Genre (e.g. advertisement, PPT) (Halliday, 1978). "Context of Culture" is the total socio-cultural background enabling and restricting specific situations (Halliday \& Hason, 1989).

In this study, the main channel of communication is user-generated videos of parent and child face-to-face interaction. The use of verbal and nonverbal semiotic resources to realize meaning is the main strategy to achieve the effective communication between participants in this kind of genre. For Field, we look at different scenarios where the communication takes places and the activities in the interaction, especially the parental acts. For Tenor, we look at what parent-child relation is constructed and how they are related.

Choice is another core mechanism for expressing meaning in Systemic Functional Linguistics. Making meaning is making choices and different choices have different meaning potential (Halliday, 2013). Choices function as a bridge between the language system and the instantiation of language. Since many linguistic principles are general semiotic principles (Jewitt et al., 2017), so the notion of "Choice" in language applies to other semiotic resources equally. When a child learns to act semiotically: to choose what to mean, and how to mean it, he or she will make a choice of his own resources including language, iconic gestures, gaze, body language and so on. For example, when realizing the refusal meaning, they may choose verbal resources like "No, I don't want it", or nonverbal resources like shaking the head, walking away, uttering the crying tune of a modal particle $E n$, etc. The following figure shows the analytical modal used to decode children's multimodal refusal (see Figure 1).

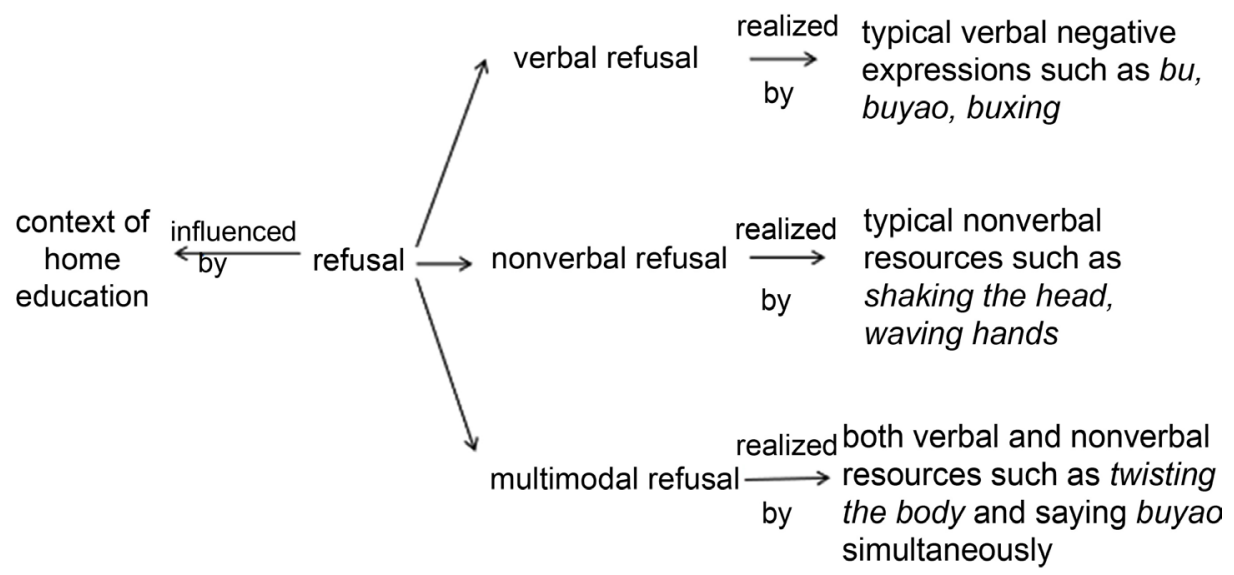

Figure 1. Analytical modal of Chinese-speaking children's refusal acts. 


\section{Corpus Collection and Annotation}

To undertake the study, researchers took video clips of naturally occurred conversations between Chinese-speaking children aged from 3 to 8 and their parents. The process of data collection lasted from November 10, 2018 to January 27, 2019, and the target children included five male children and five female children. For each child, researchers recorded 10 videos and the time length ranged from $20^{\prime \prime}$ to $4^{\prime} 11^{\prime \prime}$. The total time length of all the video data together was $115^{\prime} 17^{\prime \prime}$. After the video data were collected, researchers started to read through the raw video corpus again and again to identify all the cases of children's multimodal refusal. Then, all the multimodal refusal acts were fragmented and gathered to build a specific corpus which consisted of 110 children's multimodal acts of refusal. Next, all the 110 multimodal refusal acts were annotated and analyzed quantitatively using the multimodal discourse analyzing software $E L A N$.

\section{Acts of Parenting: A Crucial Communicative Factor}

In the context of daily parent-child communication, parenting is a crucial communicative factor which may trigger children's multimodal refusal. Therefore, a clear categorization of parental acts is both necessary and conducive to understand different forms of children's multimodal refusal from the context of home education. Analysis reveals that the parental acts in the corpus can be classified into three types according to their polarity: positive parenting (55 cases), negative parenting ( 8 cases). Besides, 47 cases of non-parenting acts (see Table 1).

\subsection{Positive Parenting}

Positive parenting in the corpus refers to cases in which parents offer suggestion, proposal or request to their children in order to cultivate either their healthy living habits or their strong communicative competence. In the corpus, children's refusal directly triggered by positive parenting occurs 55 times in total. In Example 1, child 1 was born on May, 13, 2013, Shanxi Province, China and she is a 6-year-old girl who has mastered basic language competence and can realize the refusal meaning by some negative clauses. In this example, the parent suggests her child to go to bed in view of the time, which is a positive parenting since it is healthy for children to go to bed before being late at night. The child, however, after speaking out a deferred modal particle "En" which means delay, delivers a gesture shaking his body to refuse his parent's suggestion.

Table 1. Parental acts.

\begin{tabular}{cc}
\hline Parental Acts & Frequency \\
\hline Positive parenting & 55 \\
Negative parenting & 8 \\
Non-parenting act & 47 \\
Total & 110 \\
\hline
\end{tabular}


Example 1 (video number: 20190116-01)

Parent 1: Let's go to bed.

Child 1: En..., you yourself just go to sleep. Multimodal-shaking his body left and right.

In Example 2, child 2 was born on July, 23, 2015, Shanxi Province, China and he is a 4 -year-old boy who can communicate fluently and can express refusal by some negative words and phrases. In this example, the parent suggests her child to go to pee by himself in order to train his self-care ability, which also belongs to the case of positive parenting. But the child gives his parent a multimodal refusal response, which is realized by the gesture: drag his mother's hand to his own direction.

Example 2 (video number: 20190113-02)

Parent 2: You go to pin by yourself, is that ok?

Child 2: En..., I am still a kid.

Multimodal-dragging his mother's hand to his own direction.

\subsection{Negative Parenting}

Negative parenting refers to parents' acts that are conducted for the sake of protecting children's physical and psychological health or cultivating their good characters, such as forbidding, warning and denial of children's ideas and behaviors. These parenting acts are semantically negative. There are 8 cases of this kind of multimodal refusal in total. In Example 3, child 3 was born on September, 16, 2014, Shanxi Province, China. He is a 5-year-old boy who can express himself clearly and has already mastered some negative sentential expressions. In this example, the parent explicitly forbids her child to eat cold food considering it's harmful for the health of her child's stomach, which belongs to above-mentioned negative parenting. However, the child disagrees and expresses his refusal by using verbal and nonverbal expressions continuously. His refusal starts with a negative phrase bu yao (不要), which means "will not do such". This verbal expression is accompanied by the body movement twisting the body. And then the child further says bu gei (不给), which is also a negative phrase meaning "do not give". Similarly, this second negative phrase is accompanied by a body movement lowering down his head (to avoid eye contact with his parent).

Example 3 (video number: 20190113-03)

Parent 3: You cannot eat the cold food or you will be ill.

Child 3: No, I will not give you (the cold food).

Multimodal-twisting the body, Multimodal-lowering his head.

In Example 4, child 4 was born on December, 24, 2012, Shanxi Province, China and she is a 7-year-old girl who can communicate fluently with her parents by some complete sentences. In this example, the parent imperatively commands the child not to be fastidious about the food in order to develop herself a healthy dietary habit, which also belongs to the category of negative parenting. However, the child directly refuses her parent's suggestion by using a single negative expression $b u$ (不), which is accompanied by a vector material 
process, that is, picking out the green onion out of the bowl. The combined use of verbal and nonverbal expressions in this example helps the child to conduct a refusal act against her parent's command.

Example 4 (video number: 20190116-04)

Parent 4: Swallow, gulp, gulp, gulp, don't be a picky eater.

Child 4: No.

Multimodal-picking out the green onion out of the bowl.

\subsection{Non-Parenting Act}

In parent-child communication, sometimes non-parenting acts may also trigger children's refusal, the case of which appears 47 times in the corpus. In Example 5, child 5 was born on April, 23, 2012, Shanxi Province, China and she is a 7 -year-old girl who can express refusal by some negative clauses and has possessed certain pragmatic competence. In this example, the parent delivers an offer to her daughter, that is, to apply a facial mask for her daughter, which belongs to what we call non-parenting act in this study. Instead of accepting the offer, the child refuses it by using a single negative expression bu yao (不要), which means "no". What is interesting here is that while saying bu yao, the child in the meantime smiles to her parent, which is usually thought as a positive response. In our opinion, the facial expression smiling here to a certain extent downgrades the communicative power of the negative verbal expression bu yao and prevents possible threatening of the parent's face. And this downgrading act from the child is understandable as it is quite rare for human communication to directly say no to an offer.

Example 5 (video number: 20190220-05)

Parent 5: Let me apply a facial mask for you.

Child 5: No.

Multimodal-smiling to the parent.

Similar case occurs in Example 6, in which child 6 was born on April, 23, 2012, Shanxi Province, China and she is a 7-year-old girl who can express refusal by some complete sentences and has possessed certain pragmatic competence. In this example, the parent and child are having a vacation in Sanya city where the daytime temperature is quite high. In order to prevent possible heatstroke, the parent suggests the child to put off her waistcoat, which also belongs to the above-mentioned non-parenting act. While the child refuses this suggestion through the negative expression buyao, her facial expression smiling and the gesture leaning toward her parent downgrade the communicative power of the refusal and prevent possible threatening of the parent's face.

Example 6 (video number: 2019220-06) z

Parent 6: Or you can put off your waistcoat.

Child 6: No.

Multimodal-smiling to her parent.

Multimodal-body leaning toward her parent. 


\section{The Multimodal Representational Characteristics of Chinese-Speaking Children's Refusal}

As is mentioned above, multimodal refusal refers to the realization of refusal through jointly using verbal language and nonverbal resources such as facial expression, head movement, gaze, gesture and posture, etc. This section endeavors to figure out the classification of Chinese-speaking children's multimodal refusal acts from two aspects: the primary-secondary relationship of verbal and nonverbal modals on the one hand and the degree of refusal on the other hand. By doing so, we hope to further demonstrate the representational characteristics of Chinese-speaking children's refusal and to find out the communicating habits of such group.

\subsection{Classification of Multimodal Refusal According to Modal Involvement}

According to the primary-secondary or dominant-subordinate relationship between verbal and nonverbal resources used in a single refusal act, the refusal of Chinese-speaking children can be classified into following three categories: verbal-dominant, nonverbal-dominant and verbal-nonverbal consistent refusal (see Table 2). The first category, verbal-dominant refusal refers to those refusal acts which express refusal explicitly through typical verbal negative expressions such as bu (不, no), bu yao (不要, do not do), and bu xing (不行, cannot), etc. but without the cooccurrence of typical nonverbal resources such as shaking the head, waving hands, twisting the body left and right and other left-right body movements, etc. Example 4, 5, 6 and the second part of Example 3 all belong to this type of multimodal refusal. Nonverbal-dominant refusal refers to those acts which explicitly express refusal through typical nonverbal resources mentioned above but without the cooccurrence of typical verbal negative expressions. Example 1 belongs to this type. Verbal-nonverbal consistent refusal can be further divided into two types: one is that typical verbal and nonverbal negative resources are used simultaneously, as in the first part of Example 3 where the typical verbal negative expression bu yao and the typical gestural negative form twisting the body left and right are used at the same time. The other type is that neither typical verbal nor nonverbal negative resource is used when expressing refusal, such as the case of Example 2 in which the child does not say any typical negative expression. Instead, he delivered a statement that I am still a child to implicitly refuse his parent's suggestion. Similarly, the gestural behavior of dragging

Table 2. Classification of multimodal refusal according to modal involvement.

\begin{tabular}{cc}
\hline Multimodal Refusal & Frequency \\
\hline verbal-dominant refusal & 43 \\
nonverbal-dominant refusal & 18 \\
verbal-nonverbal consistent refusal & 49 \\
Total & 110 \\
\hline
\end{tabular}


his mother's hand to his own direction is not a typical nonverbal negative resource, either.

Statistics show that there are 43 cases of verbal-dominant refusal, 18 cases of nonverbal-dominant refusal and 49 cases of verbal-nonverbal consistent refusal. Taking the type of parental acts into consideration, among those 55 cases of refusal to positive parenting, there are 20,7 and 28 cases of verbal-dominant, nonverbal-dominant and verbal-nonverbal consistent refusal. Among those 8 cases of refusal to negative parenting, there are 3,2 and 3 cases of verbal-dominant, nonverbal-dominant and verbal-nonverbal consistent refusal. Among those 47 cases of refusal to non-parenting act, there are 21, 9 and 17 cases of verbal-dominant, nonverbal-dominant and verbal-nonverbal consistent refusal.

As is revealed by the figures above, verbal-dominant refusal and verbal-nonverbal consistent refusal are equally common and much more common compared to nonverbal refusal. In other words, in parent-child communication, Chinese-speaking children conduct refusal acts multimodally or usually tend to express their refusal either verbally or verbal-nonverbally. Mono-use of nonverbal resources to express refusal is relatively uncommon.

\subsection{Classification of Multimodal Refusal According to the Degree of Refusal}

According to the semantic degree of refusal, children's multimodal refusal can be divided into two types: complete refusal on the one hand (54 cases) and partial refusal on the other hand (56 cases) (see Table 3). Among the cases of complete refusal, there are 22 cases of verbal-dominant refusal, 7 cases of nonverbal-dominant refusal and 25 cases of verbal-nonverbal consistent refusal. Among the cases of partial refusal, there are 21 cases of verbal-dominant refusal, 11 cases of nonverbal-dominant refusal and 24 cases of verbal-nonverbal consistent refusal.

As is revealed, whether it's complete refusal or partial refusal, in both situations Chinese-speaking children conduct the refusal acts through verbal expressions and the combined use of verbal and nonverbal resources more often, which proves that Chinese speaking children above the age of 3 have already acquired the competence of multimodally expressing the meaning of refusal.

Taking the types of parental acts which trigger children's refusal into consideration, among those 55 cases of refusal to positive parenting, there are 27 and 28 cases of complete refusal and partial refusal respectively, which means that there are nearly equal number of two kinds of refusal. Among those 8 cases of

Table 3. Classification of multimodal refusal acts according to the degree of refusal.

\begin{tabular}{cc}
\hline Multimodal Refusal Acts & Frequency \\
\hline Complete refusal & 54 \\
Partial refusal & 56 \\
Total & 110 \\
\hline
\end{tabular}


refusal to negative parenting, complete refusal and partial refusal appear respectively 5 and 3 times. As in those 47 cases of refusal to non-parenting acts, there are 22 and 25 cases of complete refusal and partial refusal respectively, which means that there is slightly more partial refusal than complete refusal. This result also proves that the combined use of verbal and nonverbal resources is quite common in Chinese-speaking children's refusal.

To summarize, the classification of parental acts which may trigger children's multimodal refusal and the qualitative and quantitative discussion of the multimodal representational characteristics of Chinese children's refusal acts reveal that on the one hand, while there are three kinds of parental acts that may trigger children's refusal, the number of children's multimodal refusal trigged by positive parenting and non-parenting acts is relatively close, but the number of refusal trigged by negative parenting is much smaller due to the fact that negative parenting itself does not occur frequently. On the other hand, the multimodal representation of refusal, or in other words, the combined use of verbal and nonverbal resources in expressing refusal is common in Chinese-speaking children's refusal acts.

\section{Implications for Home Education toward Chinese-Speaking Children}

Scientific home education is conducive to children's physical and psychological development and is also the footstone of school education. The findings of this study offer three aspects of implications for a more scientific home education toward Chinese-speaking children.

\subsection{Emphasizing the Multimodal Nature of Children's Refusal}

Children, especially those who are in their early childhood and have not yet fully acquired language often choose to express their intention by the combined use of verbal language and nonverbal signs such as gesture, head movement, facial expression and so on. However, neither teachers at school nor parents at home have paid enough attention to the universality of multimodal signs as children's communicative resources and to some extent have ignored children's communicative habits reflected in children's multimodal communicative acts. For instance, in Example 5 above, speaking out the negative phrase bu yao, the child meanwhile shows a facial expression of smiling to his parent, which is a nonverbal resource. As discussed, smiling in this case is an alleviation to the communicative power of the verbal refusal, decreasing the threatening of the parent's face.

Accordingly, in daily parent-child communication, parents first need to be quite aware of the fact that since it's common for children to express their intention multimodally, equal attention should be given to children's verbal and nonverbal productions to have a better understanding of their communicative intention. Second, it is beneficial for parents to consciously observe different 
contexts in which children refuse multimodally and further think about the motivation of children's multimodal refusal, summarize the characteristics of mode interaction in children's multimodal refusal. By doing so, parents are supposed to be able to understand their children's communicative intention more accurately and contribute to a more natural and harmonious parent-child communication. Finally, the analytical modal used to decode children's multimodal refusal in this paper is expected to provide a more scientific framework for the parents' understanding of their children' refusal acts in home education.

\subsection{Adjusting Parenting Acts That May Trigger Children's Refusal}

In home education, the influence of parents' acts is directly reflected in children's acts. Children's behaviors are the mapping of parents' speech and behavior (Yang, 2019). In other words, parents are the backbone of home education and the example of children's behaviors (Lin, 2005). Children's refusal to parenting acts is partly due to the reason that the act of parenting itself sometimes does not meet the expectation from the child, or it may be due to the improper expression of parenting which is not compatible with children's communicative style. Therefore, after knowing for sure the prevalence of children's multimodal refusal, parents can also appropriately adjust their parenting behaviors, especially paying attention to the use of multimodal signs rather than mono verbal language to express their intention of parenting. In daily parent-child communication, parents often use "mother talk". Considering children's common use of multimodal signs in communication, parents can adjust the expression of their mother talk by making more joint use of verbal and nonverbal expressions. Parents can add gesture, gaze and other nonverbal resources to make their mother talk more multimodal, the style of which is more in line with that of children, so that parenting will be more likely to be accepted by children.

\subsection{Cultivating Children's Ability of Multimodal Refusal}

The development of communicative competence is an important indicator of children's growth, and the development of infants and babies' action is one of the most worthwhile contents to be concerned in family upbringing (Cai \& Xie, 2017). With the advent of the era of reading images, human communication tends to be more and more multimodal. It can be predicted that multimodal communicative competence will become one of the most necessary communicative abilities for children in future, among which refusal is the most basic and common communicative behavior. There is no doubt that acquiring the ability of multimodal refusal is helpful for children to better adapt to the challenges of future multimodal social communication. Therefore, currently in home education, besides cultivating children's ability of verbally expressing refusal such as using negative phrases and rhetorical devices, parents can also consciously train their children to make better use of nonverbal signs such as gesture, head movement and facial expression, etc. to express refusal and other communica- 
tive intentions, so that children can gradually develop the ability of multiliteracy in social communication.

\section{Acknowledgements}

This research is a component of the 2018 research project "Video-corpus-based Study of Chinese-speaking Children's Multimodal Negating Act and Its Development" (18CYY022) sponsored by the National Social Science Foundation of China.

\section{Conflicts of Interest}

The authors declare no conflicts of interest regarding the publication of this paper.

\section{References}

Austin, J. L. (1979). Performative Utterance. In J. L. Austin (Ed.), Philosophical Papers 3 (pp. 233-252). Oxford: Oxford University Press.

Bressem, J., Stein, N., \& Wegener, C. (2017). Multimodal Language Use in Savosavo. Pragmatics, 2, 173-206. https://doi.org/10.1075/prag.27.2.01bre

Brown, P., \& Levinson, S. (1987). Politeness: Some Universals in Language Usage. Cambridge: Cambridge University Press. https://doi.org/10.1515/stuf-1989-0124

Cai, Y. Q., \& Xie, N. (2017). On Family Upbringing of Infants Aged 0 3 in Wuhan City and Its Improvement. Preschool Educational Research, 12, 37-48.

Chen, X., Ye, L., \& Zhang, Y. (1998). Refusing in Chinese. In G. Kasper (Ed.), Pragmatics of Chinese as Native and Target Language (pp. 119-163). Honolulu, HI: University of Hawaii Press. https://doi.org/10.1353/lan.1998.0221

Guidetti, M. (2000). Pragmatic Study of Agreement and Refusal Messages in Young French Children. Journal of Pragmatics, 5, 569-582. https://doi.org/10.1016/S0378-2166(99)00061-2

Guidetti, M. (2005). Yes Or No? How Young French Children Combine Gestures and Speech to Agree and Refuse? Journal of Child Language, 4, 911-924. https://doi.org/10.1017/S0305000905007038

Halliday, M. A. K. (1994). An Introduction to Functional Grammar. Beijing: Foreign Language Teaching and Research Press. https://doi.org/10.2307/415759

Halliday, M. A. K. (1978). Language as Social Semiotic. London: Edward Arnold Press.

Halliday, M. A. K. (2013). Meaning as Choice. In L. Fontaine, T. Bartlett, \& G. O'Grady (Eds.), Systemic Functional Linguistics: Exploring Choice (pp. 15-36). New York: Cambridge University Press.

Halliday, M. A. K., \& Hasan, R. (1989). Language, Context and Text: Aspects of Language in a Social Semiotic Perspective. Oxford: Oxford University Press.

Jewitt, C., Bezemer, J., \& O’Halloran, K. (2017). Introducing Multimodality. London: Routledge Press.

Liang B. Y. (2007). A Systemic Functional Attempt to Decode "Advances" and "Refusal" Between Strangers. Journal of Chengdu University, 1, 52-55. https://doi.org/10.1088/1126-6708/2007/01/018

Lin, Y. Q. (2005). A Brief Analysis on the Relationship between Domestic Educational 
Environment and Children's Mental Health. Family and Community Education, 4, 61-62.

Cares about the Next Generation Work Committee at Ministry of Education (2012). The Characteristics, Concepts, Researches of Domestic Education in New Era. The Status Quo, Problems and Policy Suggestion of Chinese Family (pp. 6-11). Beijing: People's Education Press.

Bressem, J., \& Müller, C. (2014). Body Language Communication: An International Handbook on Multimodality in Human Interaction. Berlin: Degruyter Mouton Press.

Petrie, A. J. (1998). Home Education and the Law. Education and the Law, 10, 123-134. https://doi.org/10.1080/0953996980100203

Searle, J. (1975). Indirect Speech Acts. In P. Cole, \& J. Morgan (Eds.), Syntax and Semantics 3: Speech Acts (pp. 59-82). New York: Academic Press.

Turnbull, W. (1992). A Conversation Approach to Explanation, with Emphasis on Politeness and Accounting. Journal of Chromatography, 1, 197-205. https://doi.org/10.1016/0021-9673(95)00824-1

Yang, Y. (2019). The Influence of Domestic Education on Children's Behavior. Educational Teaching Forum, 5, 222-223.

Zhai, B. (2016). Establishing the Domestic Education Value of the New Period. Educational Research, 3, 92-98. 